\title{
Clinical Application of Image-Guided lodine-125 Seed Implantation Therapy in Patients with Advanced Pancreatic Cancer
}

\author{
Wang Zhongmin and Chen Kemin \\ Department of Radiology, Luwan Branch Hospital, The Affiliated Hospital of Ruijin, \\ Medical School of Shanghai Jiaotong University Shanghai, \\ China
}

\section{Introduction}

Carcinoma of the pancreas is a devastating disease. The incidence of pancreatic carcinoma has increased in recent decades, yet the treatment outcome for this disease remains unsatisfactory. Despite the introduction of new therapeutic techniques and adoption of aggressive combined treatment modalities, such as external beam radiotherapy (EBRT) and chemotherapy, the prognosis of pancreatic carcinoma remained to be extremely poor, with a mortality rate of more than $90 \%$ (Geer \& Brennan, 1993). Pancreatic cancer responds poorly to surgical resection or chemotherapy, moreover only $10-20 \%$ of patients are candidates for curative surgical resection, and even with resection, long term survival still remains poor (Levin et al., 1978; Crile, 1970), therefore, the remaining patients have to seek alternative therapeutic options (Joyce et al., 1990). Biliary and gastric bypass have been used for palliation in unresectable pancreatic carcinomas and median survival in these patients was often 5-6 months (Thompson \& Nagorney, 1986; Schwarz \& Beger, 2000; Khan et al., 2010). However most of pancreatic carcinoma was diagnosed in the locally advanced or metastatic stage, both local control and management of distant metastases are the major factors that affect the prognosis of such patients. More recently, EBRT and chemotherapy have been standard adjuvants for locally advanced pancreatic carcinoma. External beam radiotherapy therapy, one of the options, is usually regarded as insensitive to pancreatic cancer and associated with more systemic side effects, although it can relieve pain in up to $50-85 \%$ of patients (Minsky et al., 1988). Also EBRT alone has failed to control disease progression and yields a median survival of 5.5-7 months (Bodner et al., 2000; Nag et al., 2006). The introduction of intraoperative electron beam radiotherapy, combined with EBRT and chemotherapy, has also failed to significantly improve long-term results, with recent studies reporting median survival rates of 7-16 months (Blasko et al., 2002; Wang et al., 2010; Cengiz et al., 2008; Monk et al., 2002). Despite the availability of many treatments, there was currently no consensus regarding the optimal therapeutic modality for unresectable pancreatic carcinomas. Therefore, it is necessary to investigate new techniques that may improve the prognosis. It has been reported that compared with external irradiation therapy, 5- Fu chemotherapy combined with radiotherapy prolonged the median survival time of patients with local advanced pancreatic cancer from 23 weeks to $42-44$ weeks 
(Mattiucci et al., Furuse et al., 2010; Kouloulias et al., 2002). However, there are few reports on interstitial implantation of radioactive seeds in the treatment of pancreatic cancer (Sun et al., 2005; Mohiuddin et al., 1992).Interstitial brachytherapy has been reported to be a useful method for local control of malignant pancreatic tumors (Takacsi et al., 2002; Enomoto et al., 2006). After placement of the radioactive seeds, the target tissue is exposed to a steady emission of gamma rays, which leads to localized ablation. Interstitial brachytherapy has been applied to unresectable pancreatic cancers in an attempt to maximize local tumor dose and minimize radiation dose to the surrounding normal structures. Therefore, radioactive iodine-125 seed implantation is another choice for treatment of malignant tumors, which is widely applied for its curative effect, minimal surgical trauma, and few complications (Ebara et al., 2008; Siegel et al., 1988; Holm et al., 1981).

\section{Advantages of biological effects of radioactive iodine-125 seed}

Advantages of ${ }^{125}$ I seeds over other forms of radiotherapy are as follows:

1. Radiation from seeds is characterized by attenuation over short distance outside the target area and low grade of depth dose, which can keep a higher accumulative dose (up to $160 \mathrm{~Gy}$ ) within the tumor. Unfortunately, serious complications such as radiation hepatitis can occur when the absorbed dose is less than $35 \mathrm{~Gy}$ as in traditional radiotherapy.

2. External radiotherapy in fractionation is only effective in cells in some phases of cell cycle. During the interval of irradiation, effect of radiotherapy is decreased because cells in the stationary phase enter into the mitotic stage. The ${ }^{125 I}$ seeds can kill tumor cells continually by keeping cells in the resting period and causing tumor stem cell apoptosis (Wang et al., 2010).

3. Deficiency of oxygen is a bottleneck of conventional external radiotherapy. Sensitive phase cells will be killed by the accumulated damaging effect of irradiation. However, cells deficient in oxygen avoid apoptosis by entering the sensitive phase.

4. Inhomogeneous radiation absorption occurs in the traditional method due to respiratory movements, which decrease the therapeutic volume. By contrast, the therapy using radioactive seeds is not affected by respiratory movements; the probability of therapeutic volume loss is obviously tiny.

5. Implantation of low energy, radioactive seeds is able to decrease the metastasis of tumor by changing the immunophenotype of tumor cells. Some research indicated that the radiobiological effect of radioactive seeds was superior to that of three dimensional conformal radiation (Mazeron et al., 2003).

\section{Indications}

1. Neutrophil leukocyte $3 \times 109 / \mathrm{L}$ or higher, platelets $70 \times 109 / \mathrm{L}$ or higher, and hemoglobin $90 \mathrm{~g} / \mathrm{L}$ or higher in peripheral blood.

2. Prothrombin index (PI) greater than $50 \%$ and partial thromboplastin time (PTT) less than 50 s; kidney function within normal range.

3. Karnofsky physical scores (KPS) greater than or equal to 60.

4. With pathologically confirmed an advanced stage of pancreatic cancer.

5. Advanced pancreatic cancer was unable to undergo open surgery due to clinical or personal reasons. 
6. Tumor maximum diameter of less than $6 \mathrm{~cm}$.

7. Previous course of chemotherapy were eligible.

\section{Contraindications}

1. Pregnant women and patients with distant metastasis (e. g. liver, lung).

2. Tumor maximum diameter greater than $6 \mathrm{~cm}$.

3. Expected survival time of less than 3 months.

4. Systemic failure.

5. Bleeding tendency.

6. Prothrombin time 3 seconds longer than the control were excluded.

7. Any previous irradiation or external radiotherapy were excluded.

\section{Preoperative preparation}

\subsection{I seed sources}

The ${ }^{125}$ I sealed seed sources were supplied by XinKe Pharmaceutical Ltd, Shanghai. For the seed implantation we used 18-G implantation needles and turntable implantation gun (XinKe Pharmaceutical Ltd, Shanghai, China). The ${ }^{125}$ I seeds were manufactured from silver rods, which absorbed ${ }^{125 I}$, and were enclosed in a titanium capsule welded by laser. The diameter of each seed was $0.8 \mathrm{~mm}$, the length was $4.5 \mathrm{~mm}$, and thickness of the wall of the titanium capsule was $0.05 \mathrm{~mm}$ (Fig.1,2). The ${ }^{125 \mathrm{I}}$ produces gamma rays $(5 \%$ of $35 \mathrm{keV}, 95 \%$ of $28 \mathrm{keV}$ ) with a half-life of 59.6 days, half-value thickness of $0.025 \mathrm{~mm}$ of lead, penetration of $17 \mathrm{~mm}$, incipient rate of $7 \mathrm{cGy} / \mathrm{h}$, a mean radioactivity of $0.694 \pm 0.021 \mathrm{mCi}(25.6 \mathrm{MBq})$, and activities of $0.5-0.9 \mathrm{mCi}$.

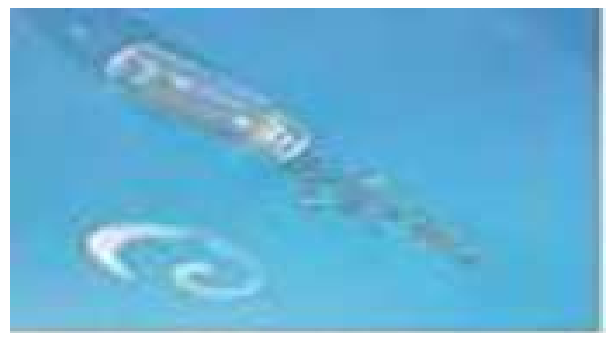

Fig. 1. Radioactive ${ }^{125}$ I seeds

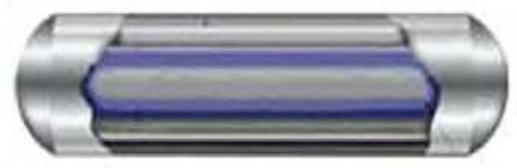

Fig. 2. Radioactive ${ }^{125}$ I seed profile chart 


\subsection{Diagnosis of pancreatic cancer}

Imaging methods were adopted for the clinical diagnosis of pancreatic cancer. Patients were first diagnosed by conventional computed tomography (CT) or magnetic resonance imaging (MRI), and by thin slice helix CT 10 days before implantation of seeds. Histological confirmation of the diagnosis was achieved by CT-guided, EUS-guided fine needle aspiration (FNA) 1 week before implantation. FNA has been accepted as a gold standard (Volmar et al., 2005; Gupta et al., 2002; Dickey et al., 1986; Mueller, 1993; Brandt et al., 1993) in the diagnosis of pancreatic cancer. Furthermore, patients had an abnormal serum CA19-9 level (higher than $37 \mathrm{U} / \mathrm{mL}, 519 \pm 439 \mathrm{U} / \mathrm{mL}$ ). All enrolled patients were diagnosed with pancreatic carcinoma before seed implantation.

\subsection{Treatment planning}

Dose distribution was calculated using a Fudan TPS2.00 brachytherapy planning system (Fudan University, Shanghai, China) based on the American Association of Physicists in Medicine TG43 brachytherapy formalism (Chen et al., 2008). The total volume of each tumor was calculated according to the CT image with the treatment planning system (TPS) before implantation (Cengiz et al., 2008). Patients underwent a detailed tumor volume study using CT scans 1-2 weeks before seed implantation. Images of each pancreatic carcinoma were obtained at $5 \mathrm{~mm}$ intervals. The radiation oncologist and surgeons together outlined the gross tumor volume (GTV) on each image and planning target volume (PTV) included GTV plus $0.5-1.0 \mathrm{~cm}$ peripheral tissue. These tracings were digitized and scanned to define the tumor volume, from which the D90 of 60-140 Gy for 125I seed irradiation, with the median of 120 Gy and the number of 125 I seeds to be implanted could be calculated. The D90 was prescribed in a way that at least $90 \%$ of the tumor volume received the reference dose. In brief, the information from CT or MRI images was reconstructed into a three-dimensional form, and the precise margin of the tumor was outlined to facilitate the calculation of tumor matched peripheral dose (MPD). The expected number of implanted seeds was calculated according to the modified level formula (Monk et al., 2002). The ${ }^{125}$ I with a nominal activity of $0.5-0.8 \mathrm{mCi} /$ seed and a diameter of less than $1 \mathrm{~mm}$ was used as a radiation source and implanted into pancreatic tumor under image guidance, at a spacing of $1 \mathrm{~cm}$.

\subsection{Calculation of the number of seeds needed for implantation}

The total volume of each tumor was calculated according to the CT image with the treatment planning system (TPS) before implantation (Bodner et al., 2000). In brief, the information from CT or MRI images was reconstructed into a three-dimensional form, and the precise margin of the tumor was outlined. An isodose curve and dose-volume histogram were drawn to concentrate the radioactivity in the target area. The expected number of implanted seeds was then calculated according to the modified Cevec formula (Monk et al., 2002) as follows:

Number of seeds needed

$\underline{1 / 2 T u m o r}$ length + width + heigth in $\mathrm{cm}] \times 5 /$ the mean activity per seed in $\mathrm{mCi}$ 3

In practice, to reach the maximum radiation effect, the number of seeds implanted was $15 \%$ more than needed. The seeds were sterilized by immersing in $2 \%$ glutaraldehyde solution for 20-30 minutes, then washed and placed into the specially designed releasing device. 
During the course of implantation, and the seeds were released and deposited in the target position.

\subsection{Pretreatment evaluation}

In most cases, the initial request for a radiotherapy consultation occurred at the time of exploratory laparotomy, so a detailed preimplant evaluation by the radiation oncologist was not possible; however, in all cases, the patients were carefully studied by the surgical team preoperatively. All patients were evaluated by comprehensive medical history, physical examination, and standard presurgical studies including complete blood count, serum chemistries, liver function tests, urine analysis, and chest X-ray, as well as the following special imaging studies: abdominal ultrasound, computerized tomography, and MRI.

\subsection{Patient preparation}

All patients signed written informed consent before the study and were informed of potential benefits and risks. The whole study protocol was approved by the Ethics Committee.

Patients fasted for $24 \mathrm{~h}$ prior to the operation, and oral laxatives were given $12 \mathrm{~h}$ before the procedure. Pancreatic secretion was inhibited by medication $24 \mathrm{~h}$ before the operation to reduce the rates of complications. For patients with jaundice, percutaneous transhepatic cholangiodrainage (PTCD) was scheduled first in order to relieve symptoms, improve liver function, and reduce the surrounding edema, after which seeds implantation was performed.

\section{Image-guided interstitial brachytherapy protocol}

\subsection{CT-guided interstitial brachytherapy protocol}

The total volume of each tumor was calculated according to the CT image with the treatment planning system (TPS) before implantation (Cengiz et al., 2008). In brief, the information from CT or MRI images was reconstructed into a three-dimensional form, and the precise margin of the tumor was outlined to facilitate the calculation of tumor matched peripheral dose (MPD). The expected number of implanted seeds was calculated according to the modified level formula (Monk et al., 2002). In practice, to reach the maximum radiation effect, the number of seeds implanted was 15\% more than needed. Implantation was guided by CT according to our TPS. The 125I with a nominal activity of 0.5-0.9 $\mathrm{mCi}$ /seed and a diameter of less than $1 \mathrm{~mm}$ was used as a radiation source and implanted into pancreatic tumor under fluoroscopy CT guidance, at a spacing of $1 \mathrm{~cm}$, avoiding puncturing vessels, pancreatic duct, and other nearby organs. Patients fasted for $24 \mathrm{~h}$ prior to the operation, and oral laxatives were given $12 \mathrm{~h}$ before the procedure. Pancreatic secretion was inhibited by medication $24 \mathrm{~h}$ before the operation to reduce the rates of complications. Sufficient breath training was given to ensure steady breath movement during the procedure. All the brachytherapy implants were performed in a standard CT room under local anesthesia. CT imaging was taken at intervals of $5 \mathrm{~mm}$. The distance between the adjacent implantation needles was approximately $1 \mathrm{~cm}$ each. Transgression of the bowel during the puncture did not result in substantial complications in our study. However, a safer approach is achieved by transversing the stomach. Intestine and colon should be avoided especially when using large-bore needles. Repeated CT with the implantation needles in place permitted adjustment of depth and angle of needle direction. 
Two to five seeds per needle were loaded, and seeds were released every 5-10 mm apart upon withdrawing the needles. For patients with jaundice, percutaneous transhepatic cholangiodrainage (PTCD) was scheduled first in order to relieve symptoms, improve liver function, and reduce the surrounding edema, after which seeds implantation was performed. Afterwards the implantation puncture site was bandaged and compressed to achieve hemostasis. Patients were kept in radiooncology/interventional ward for 4 full days.

\subsection{EUS-guided interstitial brachytherapy protocol}

All eligible patients underwent implantation of iodine-125 seeds. The operator wore a lead apron. A linear-array therapeutic echo endoscope (EG3830UT; Pentax Precision Instruments, Orangeburg, New York, USA) was inserted into the proximal stomach. The maximal diameter of the tumor was measured by real-time sector ultrasound (Olympus China Co. Ltd, Shanghai, China), with a frequency of 5-7.5 MHz. EUSwas performed to show the conformation of the pancreatic tumor and EUS images were captured by computer. The tumor volumes were calculated using EUS and CT images and 3Ddiameters of the tumors and treatment plan system software (Zhiye Medical Software Co., Shenyang, China). The minimum peripheral dose was then set to $140 \mathrm{~Gy}$ and the dose of every seed was entered into the software. The number of implants required was calculated by the software and the distribution plan maps were drawn: the experienced operator would then know the distance and direction of every target site from the center of the tumor. Iodine -125 radioactive seeds could be inserted easily through the channel of a 19-gauge therapeutic needle (Wilson-Cook Medical Inc., Win- ston-Salem, North Carolina, USA). When the needle was inserted into the target site under EUS guidance, the stylet was removed and a seed was inserted into the needle; the stylet of the needle was then advanced to push the seed forward, and the seed was released from the needle and implanted into the tissue. This implantation procedure was repeated until all the seeds were implanted into target sites according to the treatment plan. The lesion was observed by multi-slice scanning, and the relationship between the surrounding vasculature and the tumor was then identified. The puncture points and method of puncturing were determined by color Doppler technology to prevent injuring the pancreatic duct or the vasculature of the pancreas.

In principle, the seeds should be in a line and parallel to each other. The distance between each seed should be the same $(1.0 \sim 1.5 \mathrm{~cm})$. The distribution of seeds should be denser in the peripheral area so as to avoid high-dose-induced complications.

\subsection{Intraoperative ultrasound-guided interstitial brachytherapy protocol}

After the diagnosis of pancreatic cancer had been established by biopsy intraoperation, tumor volume was measured during laparotomy by intraoperative ultrasonography utilizing a megahertz linear probe. Guided by ultrasound, 18-gauge needles were implanted into mass and spaced in a parallel array at intervals of $1.0 \mathrm{~cm}$, extending at least $0.5 \sim 1 \mathrm{~cm}$ beyond the margins of the pancreatic lesions. During the placement of the needles, care was taken to avoid the needles from the pancreatic duct, small blood vessels, and the adjacent transverse colon at least $1 \mathrm{~cm}$. After needles were implanted, 125I seeds were implanted using a Mick-applicator and the spacing was maintained at $1.0 \mathrm{~cm}$ intervals. The number of 125I seeds implanted ranged from 10 to 75 , with the median number implanted of 38 . The specific activity of $125 \mathrm{I}$ ranged from 0.40 to $0.60 \mathrm{mCi}$ per seed, and the total isotope 
radioactivity implanted ranged from 4 to $37.5 \mathrm{mCi}$. An omental fat pad was placed over the implanted volume to protect the gastric and transverse colon mucosa from irradiation.

\section{Post-implant adjuvant therapy}

\subsection{Chemotherapy}

Patients who gave consent to chemotherapy received combined treatment with gemcitabine $1.0 \mathrm{~g} / \mathrm{m}^{2}$ (body surface area) and 5-fluorouracil $(5-\mathrm{Fu}) 300 \mathrm{mg} / \mathrm{m}^{2} 1$ week after the implantation. The chemotherapy was a 5- day schedule which contained gemcitabine on the first day followed by 4 days of 5-Fu. The chemotherapy was repeated every 4 weeks for up to six cycles if tolerated.

\subsection{External beam radiotherapy (EBRT)}

EBRT was generally recommended to all patients for an adjuvant aim. The patient received EBRT at 4-6 weeks after ${ }^{125}$ I seed implantation. The total doses of EBRT ranged from 35 to 50 Gy at 1.8-2.0 Gy per fraction if tolerated.

\section{Clinical benefit response (CBR)}

The clinical benefit response assessment in these patients with locally advanced pancreatic cancer was derived from the measurement of pain levels, functional impairment (assessed by the Karnofsky performance status score), and weight loss (Burris et al., 1997). For patients to achieve an overall rating of positive CBR, they had to be positive for at least one parameter (pain, performance, status, or weight) without being negative for any of the others (Hwang et al., 2004). This improvement had to last for at least 4 weeks. Patient survival, tumor responses, and the clinical benefit responses were recorded. Visual analog scale (VAS) pain score was recorded as level 0 to 10, in which 0 indicated no pain, 1 to 3 indicated mild pain, 4 to 7 meant moderate pain, and 8 to 10 severe pain. Scoring began after 125I seeds were implanted.

\section{Evaluation of curative effect}

Patients were monitored for adverse events and for abnormalities in laboratory indices, including hematological parameters, lipase, amylase, carcinoembryonic antigen (CEA), CA19-9, and liver function tests. They were assessed by physical examination (including weight, Karnofsky performance status, and visual analog scale pain score), and the tumor size was monitored by CT scan or EUS. Patients were examined by CT 2 month after the operation. The short-term efficacy was determined according to the tumor response standards suggested by the World Health Organization (Miller et al., 1981). Briefly, complete response (CR) was defined as the complete disappearance of the lesion lasting for more than 4 weeks. Partial response (PR) referred to the situation where the size (i.e., the longest dimension multiplied by maximal upright dimension) of the lesion decreased by more than $50 \%$ and then remained unchanged for 4 weeks. Stable disease (SD) was defined as the situation where the size of the tumor decreased by less than $50 \%$ or increased by less than $25 \%$. Response rate was defined as the sum of CR and PR. Local tumor control after brachytherapy was defined as the absence of tumor progression in $\mathrm{CT}(\mathrm{SD}+\mathrm{PR}+\mathrm{CR})$. The long-term efficacy included the median survival time, tumor-free survival, and 1-year 
survival rate. Serum CA19-9 level was checked every month post-implantation as an indicator of prognosis.

\section{Follow-up}

The tumor diameter, general condition, and pain score of patients were monitored and recorded during follow-up. KPS and visual analog scale (VAS) pain score were used as the main indicators of quality of life (Burris et al., 1997; Hwang et al., 2004). VAS scoring began when chemotherapy started (1 week after brachytherapy). One month after seed implantation, patients were evaluated by radiation oncologists and surgeons by physical examination, complete blood panel, chest X-ray, abdominal CT and ultrasound. One month later, a clinical consultation was provided. After that, evaluation was given every 2-3 months or sooner if a new clinical sign or symptom appeared. Time of survival was calculated from the date of diagnosis to the date of death or last follow-up. A local recurrence was defined as tumor progression (PD) within the implanted area or surrounding regions as seen on CT. Local recurrence and distant metastasis were scored until patient death and censored thereafter. The short term efficacy was determined according to the tumor response standards suggested by the World Health Organization. The long term efficacy included the median survival time, tumor-free survival, and survival rate.

\section{Complications}

The significant causes of high morbidity of 125I seed intraoperative implantation were due to the needles penetrated into pancreatic duct, small blood vessels in the pancreas and/or organ at risk resulting in fistula and abscess formation. The major long-term complication from the combined effects of multimodality treatments has been gastrointestinal bleeding and obstruction (Shipley et al., 1980).Clinical evaluation, ultrasound, and CT scans determined that the majority of patients developed metastases to the liver and peritoneal surface.

\section{Clinical status and prospects}

The survival for patients with pancreatic cancer remains poor despite standard surgical approaches, new adjuvant therapeutic techniques, and combined modality treatment. The treatment of unresectable pancreatic cancer continues to be a major challenge. More than half of patients have a locally or regionally confined tumor requiring local treatment. Resection of primary pancreatic malignancies with a curative intention is only feasible in less than $15 \%$ of all patients (Barkin \& Goldstein, 2000). Most patients will have unresectable disease even before the diagnosis is made. The median survival time for untreated patients is 4 months, and they will suffer in varying degrees from pain, anorexia, weight loss, jaundice, and intestinal obstruction (Korinthenberg et al., 2011; Jiang et al., 2010). The management of these patients is still controversial. Combined modality treatment may have a positive effect on survival and quality of life in this group of patients.

Traditional treatment for local control of advanced or metastatic pancreatic cancer involves intravenous chemotherapy with 5-fluorouracil (5-Fu) or gemcitabine; however, local recurrence and progression in the pancreas and peripancreatic lymph nodes under this treatment has been reported to be as high as $58 \%$ (Xie et al., 2006). Stereotactic radiotherapy 
(SRT) allows an escalation of radiation doses to be applied to a small target volume within a small margin. SRT is administered in one or a few fractions with the goal of sparing the surrounding normal tissue by using multiple non-coplanar field arrangements for the administration. In a phase II study on the use of SRT in the treatment of locally advanced pancreatic carcinoma by Huyer et al, the median survival time was only 5.7 months, and the one-year survival rate was 5\% (Gudjonsson, 1987). These data associate SRT with a poor outcome, unacceptable toxicity, and questionable palliative effects, making SRT unadvisable for patients with advanced pancreatic carcinoma.

In the context of multimodal oncologic therapy concepts a minimally invasive approach is often desired. Percutaneous image-guided seed implantation which can be performed without surgery or general anesthesia has attracted increasing attention because of its ability to increase radiation dose to pancreatic tumors without damaging neighboring organs (Peretz et al., 1989). With this technique, highly effective radiation doses are applied as a single fraction, ensuring protracted cell killing over a period of up to several weeks or months. Compared with other interventional procedures, advantages exist regarding interference-free and accurately predictable energy distribution, treatable size of a target lesion, and lower rate of acute adverse effects possible by maintaining tissue continuity. Extensive experiences with this technique had been collected during several preceding studies targeting liver malignancies as well as one study targeting lung malignancies (Korinthenberg et al., 2011; Jiang et al., 2010; Xie et al., 2006) 125I seed placement has become a routine treatment for malignant tumors at various sites.

In contrast, interstitial permanent implantation of radioactive seeds into the tumor site provides the advantage of delivering a high dose of irradiation to the tumor (range 140-160 Gy) which drops off sharply outside the local implanted field. ${ }^{125}$ I seeds with a half-life of approximately 59.6 days were selected as the radioactive source for permanent implantation in this study, allowing approximately $95 \%$ of the needed dose to be delivered within a year (Hoyer et al., 2005). Implantation of radioactive isotopes for the treatment of pancreatic carcinoma has been used for the past several decades. For example, Handly et al. reported the use of radium needle implantation in 7 patients for the treatment of pancreatic carcinoma in 1934 (Hilaris, 1975). Of those, one patient survived up to two years. Hilaris, who was a pioneer in the development of 125I seeds for implantation for the treatment of pancreatic carcinoma, published a study of 98 patients receiving seed implants that responded with a median survival of 7 months (Handley, 1934), with 1 patient surviving for five years. Pain control was achieved in $65 \%$ of patients and lasted between 5 and 47 months (with a median of 6 months). In a review study by Morrow et al., no difference in survival between patients treated with interstitial brachytherapy and patients treated by surgical resection at the same institution were observed (Hilaris, 1975). The median survival time was 7 months, and at least one patient survived up to five years. Pain control was achieved in $65 \%$ of the patients (Morrow et al., 1984). Syed et al. reported 18 patients treated with biliary bypass surgery, 125I interstitial brachytherapy, and EBRT (Syed et al., 1983). Ten patients with the interstitial brachytherapy were "sandwiched" between two courses of EBRT. Typically, patients received 30 Gy EBRT following biopsy and bypass surgery, then 2 weeks later an additional interstitial brachytherapy of 100-150 Gy, and then an additional 15-20 Gy EBRT was administered 3-4 weeks after interstitial implantation. The results showed a 13 month median survival time in 12 patients with head and body pancreatic carcinoma. ${ }^{125} \mathrm{I}$ seed implantation has been attempted in patients with locally advanced pancreatic carcinoma, and no difference in overall survival was found compared with the 
use of other techniques (Morrow et al., 1984). Wang et al. reported 14 patients treated with 125I seed implantation guided by intraoperative ultrasound (Wang et al., 2009). The interstitial needle position and distribution were determined using ultrasound supervision and with the intent to spare at least $1 \mathrm{~cm}$ from nearby or normal tissues including the internal pancreatic duct and small blood vessels. The placement of an omental fat pad over the implanted volume was also used to protect the gastric and transverse colon mucosa from irradiation. The result indicates that the local control of disease was achieved in $78.6 \%$ of all patients. $87.5 \%(7 / 8)$ of all patients experienced complete and partial pain relief and shown satisfactory palliative effect. The overall 1-, 2- and 3-year survival rates were $33.9 \%$, $16.9 \%$ and $7.8 \%$, respectively with the median survival of 10 months. The survival rate and survival times were found to be the most advantageous for some selected stage II/III patients.

However, there are few reports on CT-guided implantation of radioactive seeds in the treatment of pancreatic cancer. At present, the most commonly used isotope is ${ }^{125} \mathrm{I}$, and ${ }^{125} \mathrm{I}$ placement has become a routine treatment for recurrent tumors at various sites. Wang et al. reported in this group of pancreatic cancer patients (Wang et al., 2010). 31 patients implanted 125I seeds under CT guidance and yielded good local control of the disease. The results showed even distribution of the radioactive seeds with overall response rate of $61.3 \%$, local control rate of $90.3 \%$, and pain relief rate of $92 \%$.

Permanent interstitial administration of radioactive seeds appears to offer consistent and improved local control, although a major drawback is the high rate of perioperative morbidity and mortality. The significant causes of high morbidity of ${ }^{125}$ I seed intraoperative implantation were due to the needles penetrated into pancreatic duct, small blood vessels in the pancreas and/or organ at risk resulting in fistula and abscess formation. The major longterm complication from the combined effects of multimodality treatments has been gastrointestinal bleeding and obstruction (Schwarz \& Beger, 2000). The high incidence of complications maybe related to that the seeds were implanted nearby normal tissues such as gastric, colon and jejunum. The second reason may be the activity of seeds was high. The third reason maybe the doses of seeds beyond the tolerance of normal pancreas tissue. In earlier studies, perioperative mortality was $16 \%-25 \%$ from acute pancreatitis, fistulization, and abscess formation (Peretz et al., 1989). Side effects reported in the Hilaris et al., study included 1 patient developing a post-operative mortality, another patient suffered from a pancreatic fistula, 4 patients developed biliary fistula, 4 developed abscesses, 4 developed gastrointestinal bleeding, 6 developed obstruction of the gastrointestinal tract, 5 patients developed sepsis, and 4 patients developed deep venous thrombophlebitis (Handley, 1934). In comparison, the study by Syed et al. included 8 patients with a poorer prognosis, 2 patients with prolonged wound drainage, 3 patients developed insulin-dependent diabetes, and 2 patients developed other interstitial complications (Peretz et al., 1989). Also Wang et al. reported [48], one patient suffered from chylous fistula, one patient suffered from pancreatitis and one suffered from gastritis, seven patients suffered from low fever, there were no grade III and grade IV toxicity and complications, and less than most series of surgically-treated pancreatic cancer patients published in the literature (Morrow et al., 1984; Wang et al., 2009).

Local complications of advanced pancreatic carcinoma result in significant morbidity and mortality. Although systemic therapy is ultimately needed for cure, an effective locoregional therapy for the treatment of the pancreatic primary and/or regional metastases in the liver would be beneficial in patients who do not have extensive extrahepatic disease at the time of 
presentation. Current therapies, however, are of limited benefit in most patients. The high incidence of complications associated with resection of advanced pancreatic cancer and the significant gastrointestinal toxicity of external-beam radiation limit their usefulness (Yao et al., 2002). Other series of intraoperative iodine-125 implantation have been associated with mortality ranging from $0 \%$ to $16 \%$ and major morbidity of $18 \%$ (Order et al., 1996). Sun $S$ et al. reported (Sun et al., 2006), there was no significant immediate complications, such as significant bleeding or infection. The incidence of complications was 3/15 (20\%), and the adverse events were mild and not life-threatening. Although the objective response rate in patients with locally advanced pancreatic cancer was moderate, five patients experienced clinical benefit and four patients showed a partial tumor response. Pancreatic fistula is the most common complication after implantation of seeds, especially in surgery cases. In contrast, the needle used in EUS is thinner and the procedure is real-time monitored by EUS. Therefore, no pancreatic fistulae were observed in the present study.

Nevertheless there were fewer complications compared with other interventional ablation procedures. From these data it appeared that ${ }^{125} \mathrm{I}$ implantation of unresectable pancreatic tumors offered high control of the primary tumor and significant palliation of symptoms. Wang et al. reported (Wang et al., 2010), their data suggest that local control rates can be enhanced by the addition of chemotherapy. Despite lacking definitive proof, positive results allow us to continue the use of drug-seeds combination therapy. Cron et al. (Cron et al., 2005) suggested that the best time for chemotherapy is within 3-4 days after implantation of 125I seeds, because the permeability of the surrounding vasculature is promoted by the radiation effects of the seeds at that time. Wang et al. reported ten out of 31 patients in this group underwent additional chemotherapy 1 week post-treatment and tolerated it well (Wang et al., 2010). The median survival time for pure seeds implantation and drug-seeds combined therapy was 7 months and 11 months, respectively; it reached statistically significant and therefore encouraged our further evaluation. In the present study, implantation of seeds combined with chemotherapy in the treatment of pancreatic carcinoma showed preliminary effects. Although no complete remission cases were observed, the tumor progression was effectively controlled (stable disease or partial remission) in more than half of the patients (59.1\%) (Jin et al., 2008).

After promising results, we will further evaluate interventional brachytherapy as an additional tool in multimodal oncologic therapy concepts (Chen et al., 1999; Trombetta et al., 2008). This study suggested that image-guided brachytherapy using ${ }^{125}$ I seeds implantation appeared to be safe, effective, uncomplicated, and could produce adequate pain relief for treating unresectable pancreatic cancer. The present study is limited: a multimodality approach, with image guided-interstitial brachytherapy in combination with chemotherapy or external radiation, may be indicated and should be tested in further studies. Therefore, future studies should be focused on how to design a mature and feasible integrated protocol based on radioactive seeds.

\section{In conclusion}

This study suggested that image-guided brachytherapy using 125I seeds implantation appeared to be safe, effective, uncomplicated, and could produce adequate pain relief for treating unresectable pancreatic cancer. ${ }^{125} \mathrm{I}$ seed implantation with image-guided provides a satisfactory distribution of seeds in tumor mass, minimizes radiation to surrounding organs due to the sharp dose fall-off outside the implanted volume, and generates no damage (Armstrong et al., 1994). We hypothesize that a further improvement in median survival of 
patients with unresectable pancreatic carcinoma may be obtained with the combined aggressive use of EBRT, systemic chemotherapy.

\section{Examples of clinical application}
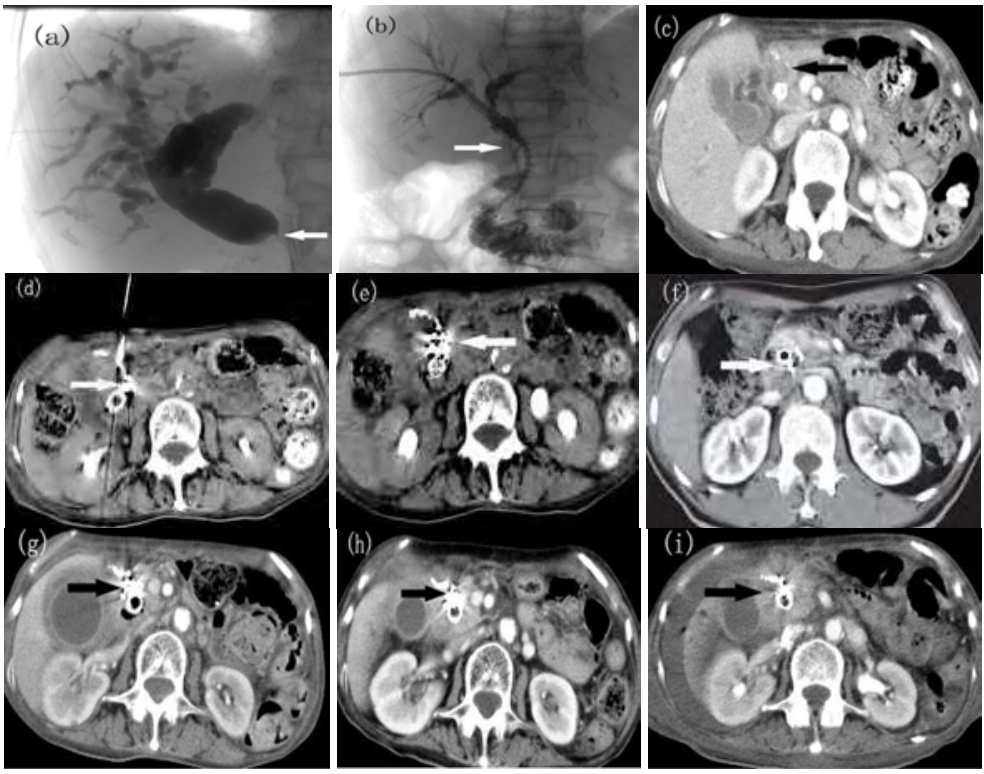

Fig. 3. 80-year-old female patient. Pancreatic carcinoma patient with ${ }^{125}$ I seeds implant under CT guidance. (a)PTCD shows the lower common bile duct stenosis (arrow), while the upper common bile duct and intrahepatic bile ducts shows expansion. (b) Common bile duct stent is implanted (arrow). (c) Contrast CT done prior to ${ }^{125}$ I seeds implantation revealed mass measured around $1.7 \times 1.8 \mathrm{~cm}$ at head of pancreas (arrow), adjacent vessels and important organs were showed clearly on the film. (d-f) Puncture needle was inserted precisely to the tumor through subcutaneous tissue under CT guidance, and $125 \mathrm{I}$ seeds were implanted (arrows). (g) 2 months follow-up. Repeated contrast CT showed reduced size of mass and aggregation of ${ }^{125}$ I seeds (arrow). (h) 4 months follow-up. Pancreatic mass showed stabilization with repeated contrast CT (arrow). (i) 6 months follow-up. Repeated contrast CT showed increased size of mass (arrow) and ascites. 

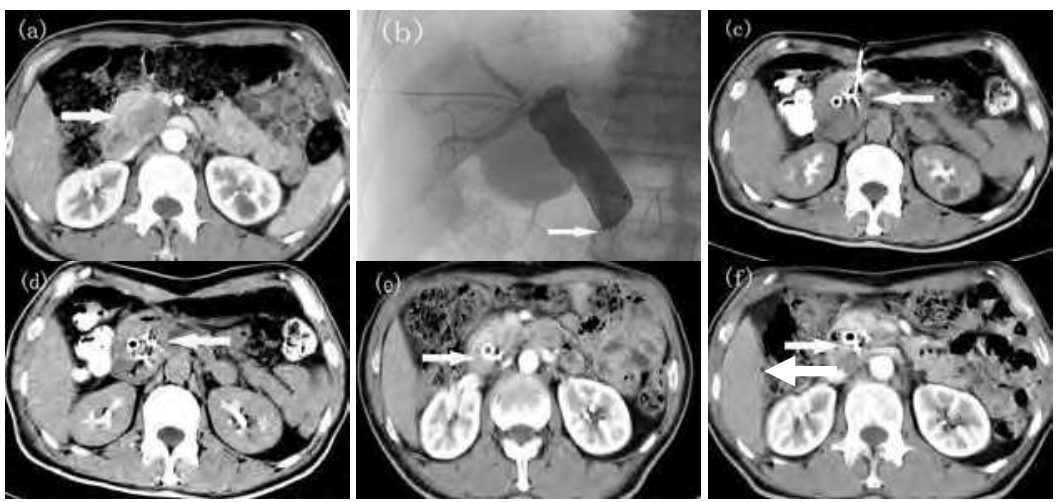

Fig. 4. 69-year-old male patient. Pancreatic carcinoma patient with ${ }^{125}$ I seeds implant under CT guidance. (a) Contrast CT done prior to ${ }^{125} \mathrm{I}$ seeds implantation revealed mass measured around $2.1 \times 2.6 \mathrm{~cm}$ at the head of pancreas (arrow), adjacent vessels and important organs were showed clearly on the film. (b) PTCD shows the lower common bile duct stenosis (arrow), while the upper common bile duct and intrahepatic bile ducts show expansion. (cd) Puncture needle was inserted precisely to the tumor through subcutaneous tissue under CT guidance, and ${ }^{125}$ I seeds were implanted (arrows). (e) 6 months follow-up. Repeated contrast CT showed reduced size of mass and aggregation of ${ }^{125}$ I seeds (arrow). (f) 12 months post ${ }^{125}$ I seed implantation, repeated contrast CT showed reduced size of mass and aggregation of 125I seeds, pancreatic mass showed stabilization (arrow).
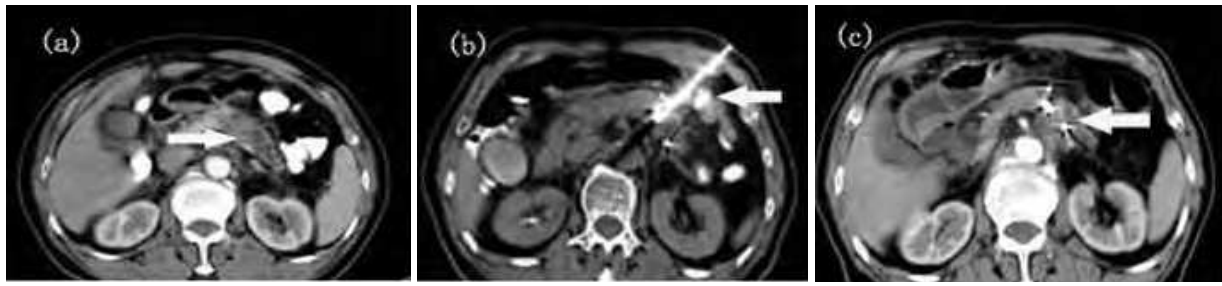

Fig. 5. 76-year-old male patient. Pancreatic carcinoma patient with ${ }^{125}$ I seeds implant under CT guidance. (a) Contrast CT done prior to ${ }^{125} \mathrm{I}$ seeds implantation revealed mass measured around $3.5 \times 5.5 \mathrm{~cm}$ at the body and tail of pancreas (arrow), adjacent vessels and important organs were showed clearly on the film. (b) Puncture needle was inserted precisely to the tumor through subcutaneous tissue under CT guidance, and ${ }^{125}$ I seeds was implanted then (arrow). (c) 2 months follow-up. Repeated contrast CT showed reduced size of mass and aggregation of 125I seeds (arrow). 

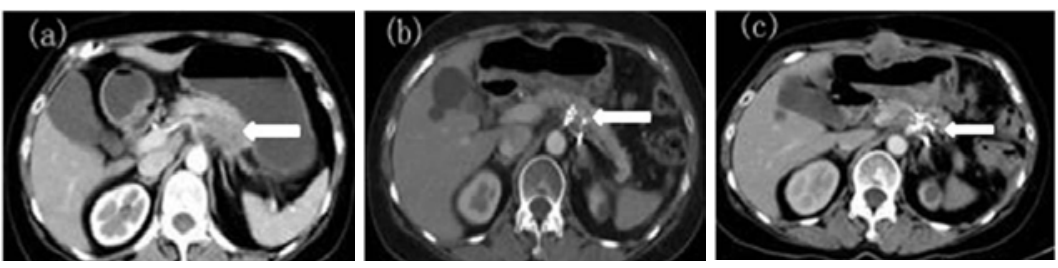

Fig. 6. 57-year-old female patient. Pancreatic carcinoma patient with ${ }^{125}$ I seeds implant under CT guidance. (a) Contrast CT done prior to ${ }^{125} \mathrm{I}$ seeds implantation revealed mass measured around $4.5 \times 5.5 \mathrm{~cm}$ at the tail of pancreas (arrow), adjacent vessels and important organs were showed clearly on the film. (b) 12 months follow-up. Repeated contrast CT showed reduced size of mass and aggregation of 125 I seeds (arrow). (c) 24 months follow-up. Repeated contrast CT showed reduced size of mass and aggregation of ${ }^{125}$ I seeds (arrow). Pancreatic mass showed stabilization.

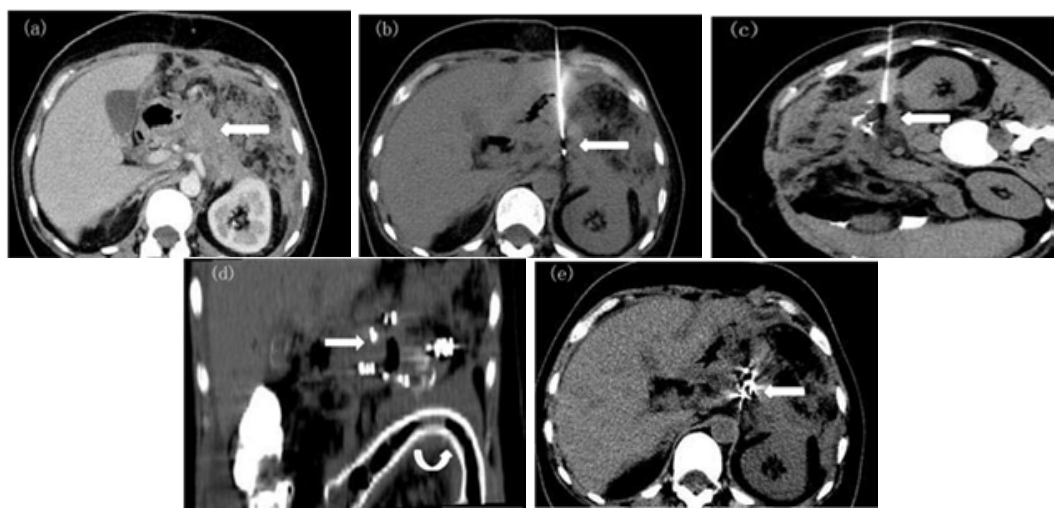

Fig. 7. 56-year-old female patient. Pancreatic carcinoma patient with ${ }^{125}$ I seeds implant under CT guidance. (a) Contrast CT done prior to ${ }^{125}$ I seeds implantation revealed mass measured around $4.5 \times 6.5 \mathrm{~cm}$ at the body and tail of pancreas (arrow), adjacent vessels and important organs were showed clearly on the film. (b) Puncture needle was inserted precisely to the tumor through subcutaneous tissue under CT guidance, and 125I seeds was implanted then (arrow). (c) Puncture needle reached precisely from prerenal space to the body and tail of pancreas cancer, which as far as possible to avoid the intestinal lumen (arrow). (d) CT 2Dimensional reconstruction after ${ }^{125}$ I seeds implantation was done to determine the distribution of ${ }^{125} \mathrm{I}$ seeds (arrow). At the same picture the duodenal stent can be seen (curved arrow). (e) 2 months follow-up. Repeated contrast CT showed reduced size of mass and aggregation of ${ }^{125}$ I seeds (arrow). 

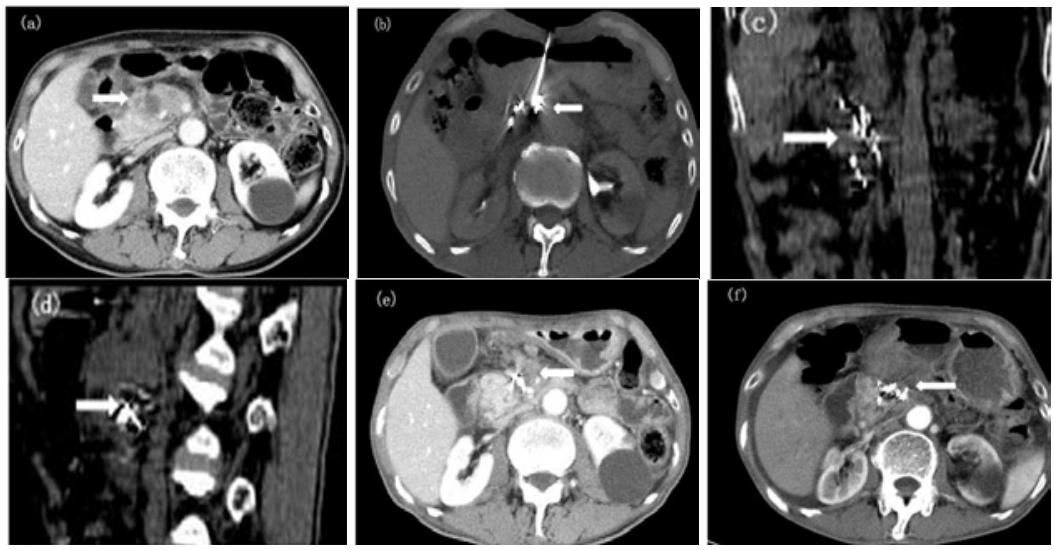

Fig. 8. 78-year-old male patient. Pancreatic carcinoma patient with ${ }^{125}$ I seeds implant under CT guidance. (a) Contrast CT done prior to ${ }^{125} \mathrm{I}$ seeds implantation revealed mass measured around $3.5 \times 4.5 \mathrm{~cm}$ at the head of pancreas (arrow), adjacent vessels and important organs were showed clearly on the film. (b) Puncture needle was inserted precisely to the tumor through subcutaneous tissue and stomach under CT guidance, and 125I seeds was implanted then (arrow). (c-d) CT 2Dimensional reconstruction after ${ }^{125}$ I implantation was done to determine the distribution of ${ }^{125}$ I seeds (arrows). (e) 2 months follow-up. Repeated contrast CT showed reduced size of mass and aggregation of ${ }^{125}$ I seeds (arrow). (f) 12 months followup. Repeated contrast CT showed reduced size of mass and aggregation of ${ }^{125}$ I seeds, pancreatic mass showed stabilization (arrow.)
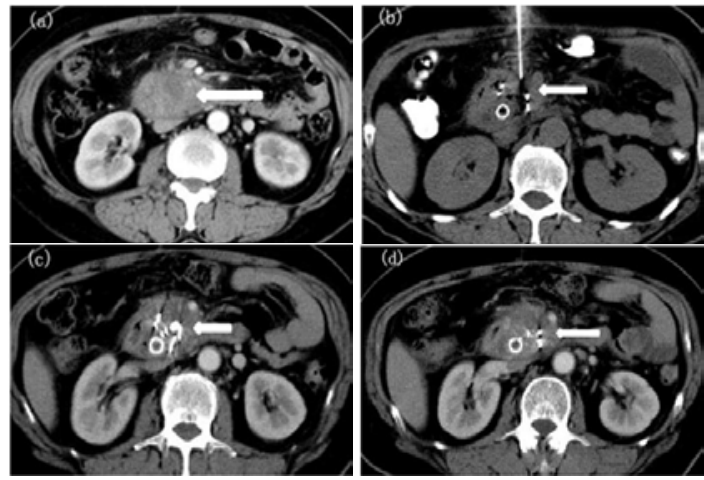

Fig. 9. 56-year-old female patient. Pancreatic carcinoma patient with ${ }^{125}$ I seeds implant under CT guidance. (a) Preoperative contrast-enhanced CT scan shows a $3.5 \mathrm{~cm} \times 4.5 \mathrm{~cm}$ tumor at the head of pancreatic carcinoma (arrow). (b) CT scan shows that 125I seeds are implanted into the tumor via $18 \mathrm{G}$ implantation needles (arrow). (c) CT scan shows the distribution of $125 \mathrm{I}$ seeds post implantation (arrow). (d) 2 months follow-up. CT scan shows pancreatic tumor partially decreased and 125I seeds gathered together (arrow). 


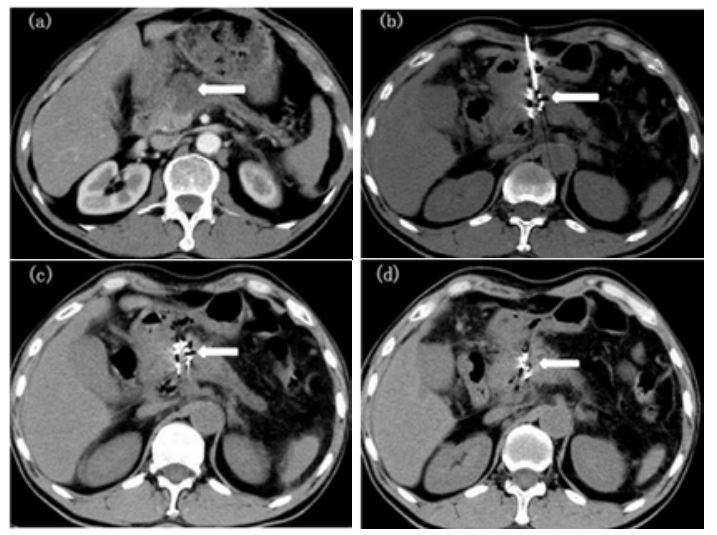

Fig. 10. 70-year-old male patient. Pancreatic carcinoma patient with ${ }^{125} \mathrm{I}$ seeds implant under CT guidance. (a) Preoperative contrast-enhanced CT scan shows a $3.0 \mathrm{~cm} \times 3.0 \mathrm{~cm}$ tumor at the body of pancreatic carcinoma (arrow). (b) CT scan shows that 125I seeds are implanted into the tumor via 18G implantation needles (arrow). (c) CT scan shows the distribution of 125I seeds post implantation (arrow). (d) 2 months follow-up. CT scan shows pancreatic tumor partially decreased and ${ }^{125}$ I seeds gathered together (arrow).

\section{References}

Armstrong, J.G.; Anderson, L.L. \& Harrison L.B. (1994). Treatment of liver metastases from colorectal cancer with radioactive implants. Cancer,Vol. 73, No. 7, Apr 1994, 1800 1804, ISSN 1097-0142.

Barkin, J.S. \& Goldstein, J.A. (2000). Diagnostic and therapeutic approach to pancreatic cancer. Biomed Pharmacother, Vol. 54, No. 7, Aug 2000, 400-409, ISSN 0753-3322.

Blasko, J.C.; Mate, T.; Sylvester J.E.; Grimm, P.D. \& Cavanagh, W. (2002). Brachytherapy for carcinoma of the prostate: techniques, patient selection, and clinical outcomes. Semin Radiat Oncol , Vol. 12, No. 1, Jan 2002, 81-94, ISSN 1053-4296.

Bodner, W.R.; Hilaris, B.S. \& Mastoras, D.A. (2000). Radiation therapy in pancreatic cancer: current practice and future trends. J Clin Gastroenterol, Vol. 30, No. 3, Apr 2000, 230 233, ISSN 0192--0790.

Brandt, K.R.; Charboneau, J.W.; Stephens, D.H.; Welch, T.J. \& Goellner, J.R. (1993). CT- and US-guided biopsy of the pancreas. Radiology, Vol. 187, No. 1, Apr 1993, 99-104, ISSN 0033-8419.

Burris, H.A.; Moore, M.J.; Andersen, J.; Green, M.R.; Rothenberg, M.L.; Modiano, M.R.; Cripps, M.C.; Portenoy, R.K.; Storniolo, A.M.; Tarassoff, P.; Nelson, R.; Dorr, F.A.; Stephens, C.D. \& Von Hoff, D.D. (1997). Improvements in survival and clinical benefit with gemcitabine as first-line therapy for patients with advanced pancreas cancer: a randomized trial. J Clin Oncol, Vol. 15, No. 6, Jun 1997, 2403-2413, ISSN 0732-183X.

Cengiz, M.; Gurdalli, S.; Selek, U.; Yildiz, F.; Saglam, Y.; Ozyar, E. \& Atahan, I.L. (2008). Effect of bladder distension on dose distribution of intracavitary brachytherapy for cervical cancer: three-dimensional computed tomography plan evaluation. Int $J$ Radiat Oncol Biol Phys, Vol. 70, No. 2, Feb 2008, 464-468, ISSN 0360-3016. 
Chen, A. ; Galloway, M.; Landreneau, R.; d'Amato, T.; Colonias, A.; Karlovits, S.; Quinn, A.; Santucci, T.; Kalnicki, S. \& Brown, D. (1999). Intraoperative 125I brachytherapyfor high-risk stage I non-small cell lung carcinoma. Int J Radait Oncol Bio Phys, Vol. 44, No. 5, Jul 1999, 1057-1063, ISSN 0360-3016.

Chen, H.H.; Jia, R.F.; Yu, L.; Zhao, M.J.; Shao, C.L. \& Cheng, W.Y. (2008). Bystander effects induced by continuous low-dose-rate 125I seeds potentiate the killing action of irradiation on human lung cancer cells in vitro. Int J Radiat Oncol Biol Phys, Vol. 72, No.5, Dec 2008, 1560-1566, ISSN 0360-3016.

Crile, G.Jr. The advantages of bypass operations over radical pancreatoduodenectomy in the treatment of pancreatic carcinoma. Surg Gynecol Obstet,Vol. 130, No. 6, Jun 1970, 1049-1053, ISSN 0039-6087.

Cron, G.O.; Beghein, N.; Crokart, N.; Chavée, E.; Bernard, S.; Vynckier, S.; Scalliet, P. \& Gallez, B. (2005). Changes in the tumor microenvironment during low-dose-rate permanent seed implantation iodine-125 brachytherapy. Int J Radiation Oncology Biol Phys, Vol. 63, No. 4, Nov 2005, 1245-1251, ISSN 0360-3016.

Dickey, J.E.; Haaga, J.R.; Stellato, T.A.; Schultz, C.L. \& Hau, T. (1986). Evaluation of computed tomography guided percutaneous biopsy of the pancreas. Surg Gynecol Obstet, Vol. 163, No. 6, Dce 1986, 497-503, ISSN 0039-6087.

Ebara, S.; Katayama, N.; Tanimoto, R.; Edamura, K.; Nose, H.; Manabe, D.; Kobayashi, T.; Kobayashi, Y.; Kobuke, M.;, Takemoto, M.; Saika, T.; Nasu, Y.; Kanazawa, S. \& Kumon, H. Iodine-125 seed implantation (permanent brachytherapy) for clinically localized prostate cancer. (2008). Acta Med Okayama, Vol. 62, No. 1, Feb 2008, 9-13, ISSN 0386-300X.

Enomoto, T.; Oda, T.; Aoyagi, Y.; Sugiura, S.; Nakajima, M.; Satake, M.; Noguchi, M. \& Ohkohchi, N. (2006). Consistent liver metastases in a rat model by portal injection of microencapsulated cancer cells. Cancer Res, Vol. 66, No. 23, Dec 2006,1113111139, ISSN 1538-7445.

Furuse, J.; Kinoshita, T.; Kawashima, M.; Ishii, H.; Nagase, M.; Konishi, M.;, Nakagohri, T.; Inoue, K.; Ogino, T.; Ikeda, H.; Maru, Y. \& Yoshino, M. (2003). Intraoperative and conformal external-beam radiation therapy with protracted 5-fluorouracil infusion in patients with locally advanced pancreatic carcinoma. Cancer, Vol. 97, No.5, Mar 2003, 1346-1352, Online ISSN 1097-0142.

Geer, R.J. \& Brennan, M.F. (1993). Prognostic indicators for survival after resection of pancreatic adenocarcinoma. Am J Surg, Vol. 165, No. 1, Jan 1993, 68-72, ISSN 01475185.

Gupta, S.; Ahrar, K.; Morello, F.A. Jr.; Wallace, M.J. \& Hicks, M.E. (2002). Masses in or around the pancreatic head: CT-guided coaxial fineneedle aspiration biopsy with a posterior transcaval approach. Radiology, Vol. 222, No. 1, Jan 2002, 63-69, ISSN 0033-8419.

Handley, W.S. (1934). Pancreatic Cancer and Its Treatment by Implanted Radium. Ann Surg,Vol. 100, No. 1, Jul 1934, 215-223, ISSN 0003-4932.

Hilaris BS. (1975). Handbook of interstitial brachytherapy, Publishing Science Group, Acton.

Holm, H.H.; Strøye, I.; Hansen, H. \& Stadil, F. (1981). Ultrasonically guided percutaneous interstitial implantation of iodine 125 seeds in cancer therapy. Br J Radiol, Vol. 54, No. 644, Aug 1981, 665-70, ISSN 0007-1285. 
Hoyer, M.; Roed, H.; Sengelov, L.; Traberg, A.; Ohlhuis, L.; Pedersen, J.; Nellemann, H.; Kiil Berthelsen, A.; Eberholst, F.; Engelholm, S.A. \& von der Maase, H. (2005). Phase-II study on stereotactic radiotherapy of locally advanced pancreatic carcinoma. Radiother Oncol, Vol. 76, No. 1, Jul 2005, 48-53, ISSN 0167-8140.

Hwang, S.S.; Scott, C.B.; Chang, V.T.; Cogswell, J.; Srinivas, S. \& Kasimis, B. (2004). Prediction of survival for advanced cancer patients by recursive partitioning analysis: role of Karnofsky performance status, quality of life, and symptom distress. Cancer Invest, Vol. 22, No. 5, 2004, 678-687, ISSN 0735-7907.

Jiang, Y.L.; Meng, N.; Wang, J.J.; Jiang, P.; Yuan, H.Sh.; Liu, C.; Qu, A. \& Yang, R.J. (2010). CT-guided iodine-125 seed permanent implantation for recurrent head and neck cancer. Radiat Oncol, Jul 2010 5: 68, ISSN (electronic) 1520-6823. http://www.ncbi.nlm.nih.gov/pubmed/20673340

Jin, Z.; Du, Y.; Li, Z.; Jiang, Y.; Chen, J. \& Liu, Y. (2008). Endoscopic ultrasonography-guided interstitial implantation of iodine 125-seeds combined with chemotherapy in the treatment of unresectable pancreatic carcinoma: a prospective pilot study. Endoscopy, Vol. 40, No. 4, Apr 2008, 314-320, ISSN 0013726X.

Joyce, F.; Burcharth, F.; Holm, H.H. \& Strøyer, I. (1990). Ultrasonically guided percutaneous implantation of iodine-125 seeds in pancreatic carcinoma. Int J Radiat Oncol Biol Phys, Vol. 19, No. 4, Oct 1990, 1049-1052, ISSN 0360-3016.

Khan, I.M.; Aurangzeb, M.; Mujeeb-Ur-Rahman \& Tayyab, M. (2010). Palliative surgery for pancreatic carcinoma. J Coll Physicians Surg Pak, Vol. 20, No. 11, Nov 2010, 719-722, ISSN 1022-386X.

Korinthenberg, R.; Neuburger, D.; Trippel, M.; Ostertag, C. \& Nikkhah, G. (2011). Longterm results of brachytherapy with temporary iodine-125 seeds in children with low-grade gliomas. Int J Radiat Oncol Biol Phys, Vol. 79, No. 4, Mar 2011, 1131-1138, ISSN 0360-3016.

Kouloulias, V.E.; Kouvaris, J.R.; Nikita, K.S.; Golematis, B.C.; Uzunoglu, N.K.; Mystakidou, K.; Papavasiliou, C. \& Vlahos, L. (2002). Intraoperative hyperthermia in conjunction with multi-schedule chemotherapy (pre-, intra- and post-operative), by-pass surgery, and post-operative radiotherapy for the management of unresectable pancreatic adenocarcinoma. Int J Hyperthermia, Vol. 18, No. 3, May-Jun 2002, 233 252, ISSN 0265-6736.

Levin, B.; ReMine, W.H.; Hermann, R.E.; Schein, P.S. \& Cohn, I.Jr. (1978). Panel: cancer of the pancreas. Am J Surg, Vol. 135, No. 2, Feb 1978, 185-191, ISSN 0147-5185.

Mattiucci, G.C.; Morganti, A.G.; Valentini, V.; Ippolito, E.; Alfieri, S.; Antinori, A.; Crucitti, A.; D'Agostino, G.R.; Di Lullo, L.; Luzi, S.; Mantini, G.; Smaniotto, D.; Doglietto, G.B.; \& Cellini, N. (2010). External beam radiotherapy plus 24-hour continuous infusion of gemcitabine in unresectable pancreatic carcinoma: long-term results of a phase II study. Int J Radiat Oncol Biol Phys, Vol. 76, No. 3, Mar 2010, 831-838, ISSN 0360-3016.

Mazeron, J.J.; Noël, G.; Simon, J.M.; Racadot, S. \& Jauffret, E. (2003). Brachytherapy in head and neck cancers. Cancer Radiother, Vol. 7, No. 1, Feb 2003, 62-72, 1278-3218.

Miller, A.B.; Hoogstraten, B.; Staquet, M. \& Winkler, A. (1981). Reporting results of cancer treatment. Cancer, Vol. 47, No. 1, Jan 1981, 207-214, Online ISSN 1097-0142. 
Minsky, B.D.; Hilaris, B. \& Fuks, Z. (1988). The role of radiation therapy in the control of pain from pancreatic carcinoma. J Pain Symptom Manage, Vol. 3, No. 4, 1988, 199205, ISSN 0885-3924.

Mohiuddin, M.; Rosato, F.; Barbot, D.; Schuricht, A.; Biermann, W \& Cantor, R. (1992). Long-term results of combined modality treatment with I-125 implantation for carcinoma of the pancreas. Int J Radiat Oncol Biol Phys, Vol.23, No. 2, 1992, 305-311, ISSN 0360-3016.

Monk, B.J.;Tewari, K.S.; Puthawala, A.A.; Syed, A.M.; Haugen, J.A. \& Burger, R.A. (2002). Treatment of recurrent gynecologic malignancies with iodine-125 permanent interstitial irradiation. Int J Radiat Oncol Biol Phys, Vol. 52, No. 3, Mar 2002, 806-815, ISSN 0360-3016.

Morrow, M.; Hilaris, B.; Brennan, M.F. (1984). Comparison of conventional surgical resection, radioactive implantation, and bypass procedures for exocrine carcinoma of the pancreas 1975-1980. Ann Surg, Vol. 199, No. 1, Jan 1984, 1-5, ISSN 0003-4932.

Mueller, P.R. (1993). Pancreatic biopsy: striving for excellence. Radiology, Vol. 187, No. 1,Apr 1993, 15-16, ISSN 0033-8419.

Nag, S.; DeHaan, M.; Scruggs, G.; Mayr, N. \& Martin, E.W. (2006). Long-term follow-up of patients of intrahepatic malignancies treated with iodine-125 brachytherapy. Int $J$ Radiat Oncol Biol Phys, Vol. 64, No. 3, Mar 2006, 736-744, ISSN 0360-3016.

Order, S.E.; Siegel, J.A.; Principato, R.; Zeiger, L.E.; Johnson, E.; Lang, P.; Lustig, R.\& Wallner, P.E. (1996). Selective tumor irradiation by infusional brachytherapy in nonresectable pancreatic cancer: a phase I study. Int J Radiat Oncol Biol Phys, Vol. 36, No. 5, Dec 1996, 1117-1126, ISSN 0360-3016.

Peretz, T.; Nori, D.; Hilaris, B.; Manolatos, S.; Linares, L.; Harrison, L.; Anderson, L.L.; Fuks, Z. \& Brennan, M.F. (1989). Treatment of primary unresectable carcinoma of the pancreas with I-125 implantation. Int J Radiat Oncol Biol Phys, Vol. 17, No. 5, Nov 1989, 931-935, ISSN 0360-3016.

Schwarz, A.; Beger, H.G. (2000). Biliary and gastric bypass or stenting in nonresectable periampullary cancer: analysis on the basis of controlled trials. Int J Pancreatol, Vol.27, No. 1, Feb 2000, 51-58, ISSN 0169-4197.

Shipley, W.U.; Nardi, G.L.; Cohen, A.M. \& Ling, C.C. (1980). Iodine-125 implant and external beam irradiation in patients with localized pancreatic carcinoma: a comparative study to surgical resection. Cancer, Vol. 45, No. 4, Feb 1980, 709-714, Online ISSN 1097-0142.

Siegel, J.H.; Lichtenstein, J.L.; Pullano, W.E.; Ramsey, W.H.; Rosenbaum, A,; Hhlpern, G.; Nonkin, R. \& Jacob, H. (1988). Treatment of malignant biliary obstruction by endoscopic implantation of iridium 192 using a new double lumen endoprosthesis. Gastrointest Endosc, Vol. 34, No. 4, Jul-Aug 1988, 301-306, ISSN 0016-5107.

Sun, S.; Qingjie, L.; Qiyong, G.; Mengchun, W.; Bo, Q. \& Hong, X. (2005). EUS-guided interstitial brachytherapy of the Pancreas: a feasibility study. Gastrointest Endosc, Vol. 62, No. 5, Nov 2005, 775-779, ISSN 0016-5107.

Sun, S.; Xu, H.; Xin, J.; Liu, J.; Guo, Q. \& Li, S. (2006). Endoscopic ultrasound-guided interstitial brachytherapy of unresectable pancreatic cancer: results of a pilot trial. Endoscopy, Vol. 38, No. 4, Apr 2006, 399- 403, ISSN 0013726X. 
Syed, A.M.; Puthawala, A.A. \& Neblett, D.L. (1983). Interstitial iodine-125 implant in the management of unresectable pancreatic carcinoma. Cancer,Vol. 52, No. 5, Sep 1983, 808-813, Online ISSN 1097-0142.

Takácsi-Nagy, Z.; Varga, J.; Poller, I.; Fodor, J.; Polgár, C.; Petrányi, A.; Major, T. \& Németh, G. (2002). Successful treatment of a T1 cancer of the pancreatic head with high dose rate brachytherapy and external radiotherapy. Hepato-gastroenterology, Vol. 49, No. 45, May-Jun 2002, 844-846, ISSN 0172-6390.

Thompson, E. \& Nagorney, D.M. (1986). Stapled cholecystojejunostomy and gastrojejunostomy for the palliation of unresectable pancreatic carcinoma. Am J Surg, Vol. 151, No. 4, Apr 1986, 509-511, ISSN 0147-5185.

Trombetta, M.G.; Colonias, A.; Makishi, D.; Keenan, R,.;Werts, E.D.; Landreneau, R. \& Parda, D.S. (2008). Tolerance of the aorta using intraoperative iodine-125 interstitial brachytherapy in cancer of the lung. Brachytherapy, Vol. 7, No. 1, Jan-Mar 2008, 5054, ISSN 1538-4721.

Volmar, K.E.; Vollmer, R.T.; Jowell, P.S.; Nelson, R.C. \& Xie, H.B. (2005). Pancreatic FNA in 1000 cases: a comparison of imaging modalities. Gastrointest Endosc, Vol. 61, No. 7, Jun 2005, 854-861, ISSN 0016-5107.

Wang, J.J.; Jiang, Y.L.; Li, J.N.; Tian, S.Q.; Ran, W.Q. \& Xiu, D.R. (2009). Intraoperative ultrasound-guided iodine-125 seed implantation for unresectable pancreatic carcinoma. J Exp Clin Cancer Res, Vol. 28, Jun 2009, 88, ISSN 1756-9966.

Wang, J.J.; Yuan, H.S.; Li, J.N.; Jiang, Y.L.; Tian, S.Q. \& Yang, R.J. (2010). CT-guided radioactive seed implantation for recurrent rectal carcinoma after multiple therapy. Med Oncol, Vol. 27, No. 2, Jun 2010,421-429, ISSN 1357-0560.

Wang, Z.M.; Liu, Y.; Liu, F.J.; Chen, K.M. \& Huang, G. (2010). Clinical efficacy of CT-guided iodine-125 seed implantation therapy in patients with advanced pancreatic cancer. Eur Radiol, Vol. 20, No. 7, Jul 2010, 1786-1791, ISSN 0938-7994.

Xie, D.R.; Liang, H.L.; Wang, Y.; Guo, S.S. \& Yang, Q. (2006). Meta-analysis on inoperable pancreatic cancer: a comparison between gemcitabine-based combination therapy and gemcitabine alone. World J Gastroenterol, Vol. 12, No. 43, Nov 2006, 6973-6981, ISSN 1007-9327.

Yeo, C.J.; Cameron, J.L.; Lillemoe, K.D.; Sohn, T.A.; Campbell, K.A.; Sauter, P.K.; Coleman, J.; Abrams, R.A. \& Hruban, R.H. (2002). Pancreaticoduodenectomy with or without distal gastrectomy and extended retroperitoneal lympha-denectomy for periampullary adenocarcinoma, part 2: randomized controlled trial evaluating survival, morbidity, and mortality. Ann Surg, Vol. 236, No. 3, Sep 2002, 355-368, discussion 366-368, ISSN 0003-4932. 


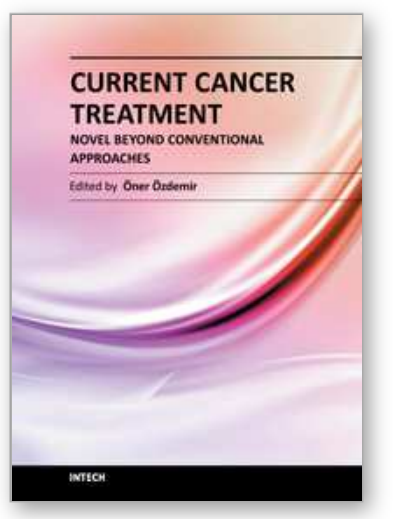

\author{
Current Cancer Treatment - Novel Beyond Conventional \\ Approaches \\ Edited by Prof. Oner Ozdemir
}

ISBN 978-953-307-397-2

Hard cover, 810 pages

Publisher InTech

Published online 09, December, 2011

Published in print edition December, 2011

Currently there have been many armamentaria to be used in cancer treatment. This indeed indicates that the final treatment has not yet been found. It seems this will take a long period of time to achieve. Thus, cancer treatment in general still seems to need new and more effective approaches. The book "Current Cancer Treatment - Novel Beyond Conventional Approaches", consisting of 33 chapters, will help get us physicians as well as patients enlightened with new research and developments in this area. This book is a valuable contribution to this area mentioning various modalities in cancer treatment such as some rare classic treatment approaches: treatment of metastatic liver disease of colorectal origin, radiation treatment of skull and spine chordoma, changing the face of adjuvant therapy for early breast cancer; new therapeutic approaches of old techniques: laser-driven radiation therapy, laser photo-chemotherapy, new approaches targeting androgen receptor and many more emerging techniques.

\title{
How to reference
}

In order to correctly reference this scholarly work, feel free to copy and paste the following:

Wang Zhongmin and Chen Kemin (2011). Clinical Application of Image-Guided lodine-125 Seed Implantation Therapy in Patients with Advanced Pancreatic Cancer, Current Cancer Treatment - Novel Beyond Conventional Approaches, Prof. Oner Ozdemir (Ed.), ISBN: 978-953-307-397-2, InTech, Available from: http://www.intechopen.com/books/current-cancer-treatment-novel-beyond-conventional-approaches/clinicalapplication-of-image-guided-iodine-125-seed-implantation-therapy-in-patients-with-advanced-

\section{INTECH}

open science | open minds

\section{InTech Europe}

University Campus STeP Ri

Slavka Krautzeka 83/A

51000 Rijeka, Croatia

Phone: +385 (51) 770447

Fax: +385 (51) 686166

www.intechopen.com

\section{InTech China}

Unit 405, Office Block, Hotel Equatorial Shanghai

No.65, Yan An Road (West), Shanghai, 200040, China

中国上海市延安西路65号上海国际贵都大饭店办公楼 405 单元

Phone: +86-21-62489820

Fax: $+86-21-62489821$ 
(C) 2011 The Author(s). Licensee IntechOpen. This is an open access article distributed under the terms of the Creative Commons Attribution 3.0 License, which permits unrestricted use, distribution, and reproduction in any medium, provided the original work is properly cited. 